\title{
Design, Fabrication, Analysis, and Testing of a Micro Air Vehicle Propeller
}

\author{
Adam Smedresman, ${ }^{*}$ Derrick Yeo, ${ }^{\dagger}$ and Wei Shyy ${ }^{\ddagger}$ \\ Department of Aerospace Engineering, University of Michigan, Ann Arbor, Michigan, 48109
}

\begin{abstract}
Propellers are critical for the performance of fixed wing micro air vehicles (MAVs). Due to the low Reynolds number nature of MAV flight, the established propeller design practice needs to be reexamined. Experimental data is required to validate predictions; however, few studies have been conducted because the small dimensions and forces of the propellers make fabrication and reliable testing extremely challenging. The present study describes the development of a fabrication technique and a test stand, and the use of aerodynamic analyses to assess the performance of small fixed pitch propellers. Wind tunnel data for a single prototype propeller showed lower thrust, torque, and efficiency than predicted by commercially available vortex theory codes. Potential explanations for these discrepancies include inaccuracy of the airfoil coefficients, error resulting from approximations in the vortex theory, and inaccuracies related to fabrication.
\end{abstract}
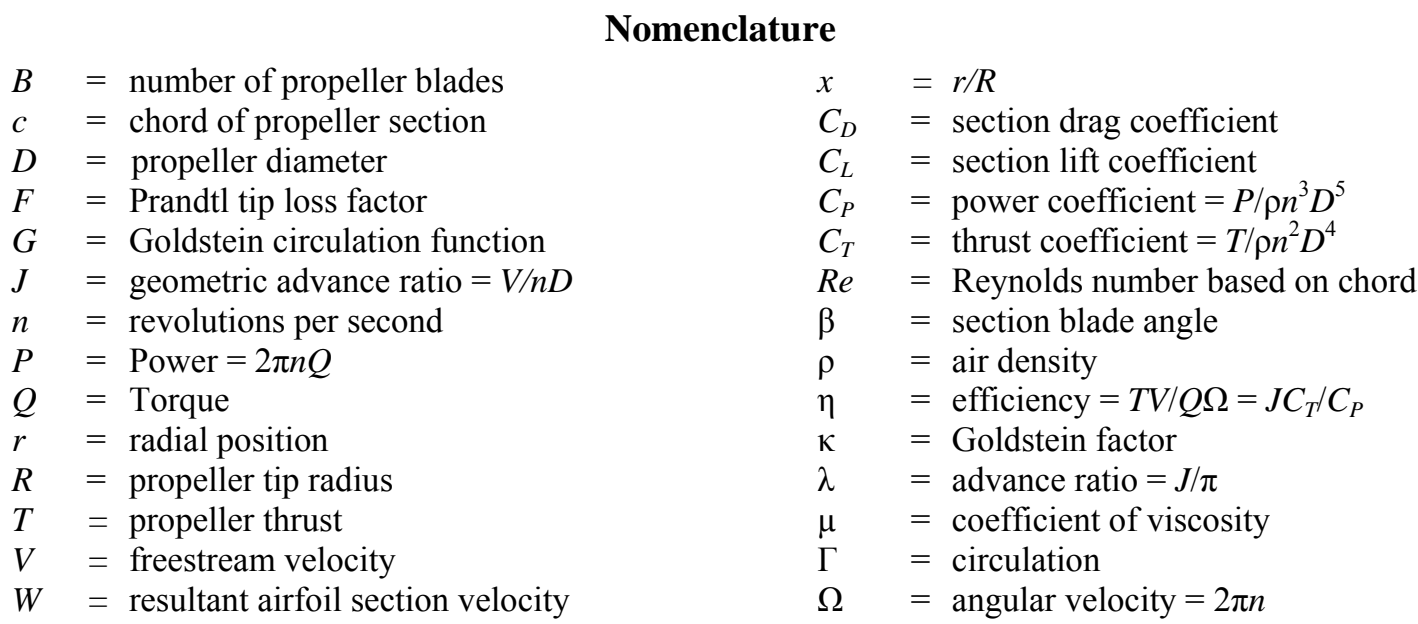

\section{Introduction}

$\mathrm{M}$ icro Air Vehicles (MAVs) have been the subject of increased attention for the past decade because of their potential unique military and civilian applications. ${ }^{1-3}$ These include surveillance, search and rescue, and remote detection. Originally, MAVs were envisioned with wing spans of $15 \mathrm{~cm}$ or less and flight speeds of 30-60 $\mathrm{km} / \mathrm{h}$. As pointed out by Shyy et al., ${ }^{3}$ MAV flight has two prominent features: (i) low Reynolds number, resulting in unfavorable aerodynamic conditions compared to passenger-size flight vehicles, and (ii) small physical dimensions, resulting in certain favorable scaling characteristics including structural strength, reduced speed of stall, and low inertia. Since then, substantial progress has been made. However, there still remain great challenges in the fields of controls, propulsion, and aerodynamics. This is especially true as MAVs continue to decrease in size, while performance expectations increase.

\footnotetext{
* Graduate Student, 1320 Beal Ave., AIAA Student Member.

${ }^{\dagger}$ Doctoral Candidate, 1320 Beal Ave., AIAA Student Member.

* Clarence L. "Kelly" Johnson Collegiate Professor, AIAA Fellow; currently, Provost \& Chair Professor, Mechanical Engineering, The Hong Kong University of Science and Technology, Hong Kong.
} 
The aerodynamics of MAVs present challenges due to the low Reynolds number regime in which they operate. Typical chord-based Reynolds numbers range between $10^{4}$ and $10^{5}$. MAVs are subject to unfavorable flight characteristics such as sensitivity to transition, separation, and reduced lift-to-drag ratios. Because high endurance is desired and lift is at a premium, propulsive efficiency is a top priority. With the increased use of MAVs, there has been growing interest in validating propeller performance for fixed wing configurations and improving the design practices at low Reynolds numbers.

Early fixed wing MAVs often used commercially available propellers because of their convenience, but peak efficiencies were sometimes as low as $30 \%{ }^{4-6}$ It was discovered that efficiency could be enhanced by modifying the geometries of these propellers, but these efforts did not lead to significant improvements in the design process. Of the limited studies on MAV propeller design in the literature, most use a vortex theory-based minimum induced loss (MIL) optimization. Because of limited experiments for validation, questions remain about the applicability and details of these theories, which may differ significantly in their implementation.

Another persisting question is the validity of 2-D airfoil data in these propeller models, especially after stall. As Larrabee $^{7}$ stated, "blade element boundary layers exhibit radial as well as chordwise growth," and that assuming simple two-dimensional section characteristics was "naïve to say the least." The goal of the present study was to design a propeller using a minimum induced loss methodology and to experimentally test it for validation of predictions.

\section{Background}

Propeller theory dates back to Rankine's 1865 study of marine propellers.8 His theory modeled the fluid momentum equations of an 'actuator disk,' or a rotor with an infinite number of blades. In $1878 \mathrm{~W}$. Froude introduced blade element theory, which considered a propeller's geometry and modeled the blade as the sum of independent two-dimensional airfoil sections. ${ }^{9}$ Further progress was made in the early $20^{\text {th }}$ century when Prandtl's wing lifting line theory introduced the concept of the vortex sheet. This was applied to propellers to optimize and predict performance. Classical vortex theory was developed by Betz, ${ }^{10}$ Prandtl, ${ }^{11}$ Goldstein, ${ }^{12}$ Theodorsen, ${ }^{12}$ and others. In 1919, Betz identified the conditions for minimum induced loss, known as the "Betz condition," which calls for a prescribed "rigid" vortex sheet of constant pitch in the trailing wake of the propeller. Prandtl developed a closed-form approximation for the flow around this helicoidal wake based on two-dimensional flow. This approximation, called the $F$ factor, is good at low advance ratios and improves with an increasing number of blades. ${ }^{14}$ In 1929, Goldstein found the exact solution to the flow field and circulation distribution for the minimum induced loss condition, assuming that the induced velocity is normal to the resultant velocity. ${ }^{14}$ The result is the analogue to a minimum induced loss elliptically loaded wing. An analytical solution has not been found but numerical results are available in tabulated form. Goldstein's approach was limited to lightly loaded propellers although a correction for moderately loaded propellers was offered. ${ }^{12}$ Theodorsen re-applied the Goldstein function and showed that the lightly loaded requirement could be removed by examining the vortex sheets at a distance far from the propeller. ${ }^{12}$ Recent refinements of classical vortex theory include the compensation of blade angle of attack and lift coefficient for curvature and finite blade thickness. ${ }^{14}$

In one of the first investigations of MAV propeller design, Grasmeyer and Keennon optimized propellers for the "Black Widow" MAV with a minimum induced loss methodology of Adkins and Liebeck., ${ }^{4,15}$ It is estimated that the propellers operated at a blade Reynolds number between $2 \times 10^{4}$ and $3 \times 10^{4}$ at $75 \%$ radius. Here, Reynolds number is defined using the chord length and the resultant velocity of the airfoil section. The propellers were designed for a loiter mission profile with a multi-disciplinary optimization code which modeled battery, motor, and aerodynamic performance. Grasmeyer and Keennon reported that commercially available propellers, even when modified, did not meet the needs for the Black Widow, so they built their own out of composites. In wind tunnel experiments, the propellers performed in close agreement with their predictions. One of their propellers reached a peak efficiency of greater than $80 \%$, which was surprising given that many hobby propellers of much larger size do not achieve $60 \%$ efficiency ${ }^{16}$ These findings support the use of vortex theory as a design tool for propellers at the MAV scale. However, little is known about the airfoil geometries of Grasmeyer and Keennon's propellers or the airfoil data that was used in the predictions.

Grasmeyer and Keennon's report also highlighted some of the tradeoffs that are made in MAV design. They reported that overall propulsive efficiency could be improved slightly at the expense of propeller efficiency by designing the propeller for an electric motor with a fast direct drive, due to reduced mass and thrust requirements as compared to using a gearbox and a higher advance ratio propeller. In their final design, Grasmeyer and Keennon chose a propeller with an advance ratio of $J=0.44$ and an operating efficiency of $73 \%$ over a propeller with an advance ratio of $J=1.3$ and an operating efficiency of $78 \%$. 
Influenced by Grasmeyer and Keennon, Lee et al. ${ }^{17}$ optimized a MAV propeller geometry based on the same design conditions as the Black Widow. They implemented a numerical optimization code and used a response surface methodology (RSM) to maximize efficiency, which was also predicted using the Adkins and Liebeck method. In their optimization algorithm, constraints included propeller thrust, tip Mach number (to limit drag from compressibility effects) and activity factor, to characterize the power absorbed by the propeller. The algorithm additionally incorporated two-dimensional airfoil characteristics obtained using XFOIL, ${ }^{18}$ a 2-D viscous panel code. Spanwise two-dimensional airfoil shapes were optimized for maximum lift-to-drag ratio. A Monte Carlo simulation and Chebyshev inequality were used to converge the design space and obtain their design. The final design had a predicted efficiency within a few percent of the Black Widow propeller's experimentally measured efficiency. Lee et al. predicted that their airfoil optimization increased overall efficiency by $3.4 \%$ compared to using a constant low Reynolds number airfoil. This demonstrates the sensitivity of airfoil performance across a range of Reynolds numbers on the propeller blade. The appreciable performance gains and the closeness in the predicted efficiency to the Black Widow design suggests that Grasmeyer and Keennon also used optimized airfoil sections.

Youngren and Chang ${ }^{19}$ designed and fabricated a $10.2 \mathrm{~cm}$ propeller for a mono-wing rotorcraft MAV. Using XROTOR, ${ }^{20}$ a code developed at MIT, they designed a propeller for minimum induced loss, but with modifications such as a squared-off tip to reduce low Reynolds number drag. This design choice represents a compromise between induced and profile losses. The propeller was operating at a chord-based Reynolds number that ranged between $1.5 \times 10^{4}$ and $4.8 \times 10^{4}$. Special airfoils were designed using XFOIL for laminar flow at low lift coefficients. A 3-D laminar flow numerical simulation of the propeller was implemented. Flow on the blade was found to be largely two-dimensional, which provides an argument for the use of 2-D airfoil data in the model. The CFD simulation showed that the new design had reduced hub separation and more evenly distributed tip vortices as compared to a commercial propeller. Peak efficiency of the propeller design was predicted to be $76 \%$ from the numerical simulation. XROTOR predictions for a band of profile drag values were similar. Wind tunnel tests of the fabricated propeller showed efficiencies that were about $10 \%$ lower, but because torque was not measured, a model for the motor efficiency was introduced to estimate this value. Interestingly, the thrust at the design point was found to be significantly higher than that predicted by XROTOR.

Moffitt et al. ${ }^{21}$ tested unmanned aerial vehicle (UAV) size propellers between 16 and 22 inches in diameter to compare to predictions from vortex theory. Reynolds numbers were in the range of $1 \times 10^{5}$. The blades were cut at many stations and their chord and twist distributions were measured. Airfoil sections were then digitized and analyzed using XFOIL. An important aspect of their study was comparing the predictions from vortex theory when using two radial momentum loss factors: the $F$ factor and the Goldstein circulation function. It was found that both formulations captured the trends from the experiment well. At lower advance ratios agreement worsened, which was thought to be due to the stalling of the blades which can introduce highly three-dimensional separation. At these low advance ratios, the $F$ factor formulation predicted the thrust and torque trends slightly better. Moffitt et al. also conducted a system sensitivity analysis of the measurement and modeling uncertainties on the propeller performance. They found that the largest source of uncertainty in their predictive model was the 2-D airfoil section data.

\section{Theory}

The present study applied the codes QMIL and QProp, which were written by Mark Drela. ${ }^{22}$ QMIL is a code for designing a propeller of minimum induced loss (MIL) for user-inputted design parameters. QProp predicts offdesign propeller performance, when the Betz condition no longer applies. QMIL uses an extended Larrabee ${ }^{7}$ method which uses a lifting line representation of the propeller with relations from classic vortex theory and blade element theory (see Ref. 22 for further details). In determining "tip losses," a local wake advance ratio instead of the propeller advance ratio is used. This gives improved predictions at increasing disk loading where the kinematic advance ratio of the propeller and that of the wake may differ significantly. It should be noted that when designing efficient propellers, heavy thrust loadings are to be avoided because efficiency drops with increased loading. As Larrabee notes, "propellers of high efficiency must have values of $C_{P}\left(\right.$ and $C_{T}$ ) below 0.5 anyway."

One detail of QMIL theory worthy of discussion is the choice of momentum loss factor, which accounts for radial flow of the fluid on a propeller blade. From Stokes' law, using the notation of Drela, the circumferentiallyaveraged tangential component of the induced velocity can be related to the bound circulation $\Gamma$ of the blades from the Kutta-Joukowsky theorem at each radius $r$ by

$$
B \Gamma=4 \pi r \bar{v}_{t}
$$

where local circulation $\Gamma$ can be expressed as 


$$
\Gamma=\frac{1}{2} W c C_{L}
$$

At this stage an empirical relation is used by Drela to relate the circumferentially-averaged tangential velocity to the tangential velocity at the blade, $v_{t}$,

$$
\bar{v}_{t}=v_{t} F \sqrt{1+\left(4 \lambda_{w} R / \pi B r\right)^{2}}
$$

where $\lambda_{w}$ is the advance ratio of the wake. Using Eq. (3), Prandtl's momentum loss factor $F$ is applied to account for the radial flow of the propeller that meets the Betz condition. It is used as an alternative to Goldstein's "exact" solution using his proportionality factor $\kappa$, which cannot be solved analytically but is available in different forms in tabulated charts for varying advance ratios and number of blades. ${ }^{23-25}$ The $F$ factor was derived from twodimensional flow past an infinite rectangular grid and is accurate at low advance ratios and with a large number of blades. ${ }^{26}$ It is a convenient alternative because of its simple closed form solution, given in Eq. (4).

$$
F=\frac{2}{\pi} \cos ^{-1} \exp \left[-\left(\frac{B}{2 \lambda_{w}}\right)(1-x)\right]
$$

By combining Eqs. (1) and (3), a relation between the local circulation and the tangential velocity of the propeller blade can be made. The axial component of induced velocity can then be solved for using the assumption that the induced velocity is normal to the resultant velocity at the propeller.

When these relations are used as a design tool for a MIL propeller, it can be seen that the momentum loss factor plays a role in calculating circulation and blade chords. A graphical comparison between formulations using the Goldstein factor and the $F$ factor can be made by plotting $G(x)$, the non-dimensionalized circulation distribution. This is shown in Fig. 1, which was reproduced from a figure by Larrabee and French. ${ }^{27}$ The figure shows that agreement between the circulation distributions using the $F$ factor and the Goldstein factor is good at low advance ratios and with a large number of blades. ${ }^{22}$ However, for a two-bladed propeller at an advance ratio of $\lambda=0.5$ there is significant disagreement between the two formulations. Prandtl's $F$ factor underestimates circulation near the root and overestimates circulation near the outer half of the blade. According to Bauer, ${ }^{28}$ using the $F$ factor in the Adkins and Liebeck MIL method can overestimate propeller efficiency because it is a component in its calculation, and only accounts for circumferential and axial losses but not for 3-D energy losses in the wake. (Interestingly, Moffitt et al. ${ }^{21}$ found

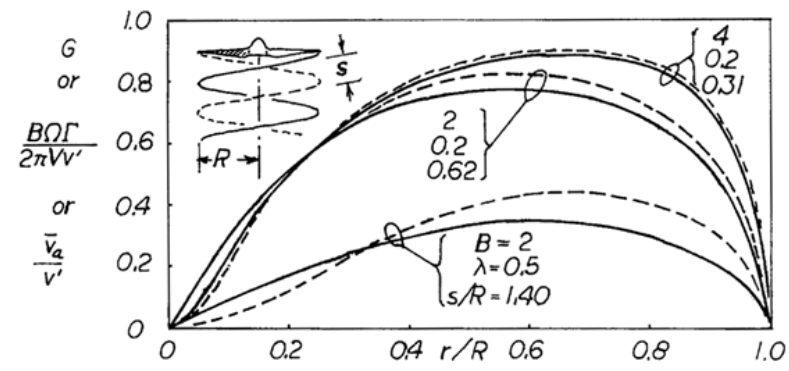

Figure 1. Comparison of circulation distributions calculated from Goldstein (solid line) and the Prandtl $F$ factor (dashed line). Plot reproduced from Ref. 27.

that both the $F$ factor and the Goldstein distribution underestimated efficiency in predictions for off-design performance of an arbitrary UAV-size propeller). Bauer also mentions a difference in blade twist when compared to using the method of Theodorsen, which applies the Goldstein function.

The discrepancies discussed above may be more relevant for MAVs than for full-scale aircraft, due to inherent design constraints. For example, in many cases it would be undesirable to have more than two blades on a MAV. Also, at low Reynolds numbers, a high advance ratio propeller will still have greater peak efficiency than a low advance ratio propeller. The highest efficiency Black Widow propeller, for instance, had an advance ratio $J=1.3(\lambda$ $=0.41$ ) and was two-bladed. Sensitivity analysis of propeller performance to details such as the momentum loss factor in vortex theory has not been studied, and may merit further investigation. In any case, it is an issue to be aware of in propeller design.

\section{Prototype Propeller Design}

The propeller prototype used in this study was designed in order to conduct a preliminary test of the validity of vortex theory at the MAV scale. Emphasis was placed on creating an efficient propeller in the design space for a MAV, not on designing for a particular vehicle. In this study the parameters chosen were for a vehicle slightly lighter and slower-flying than the Black Widow, a well-documented MAV (see Ref. 4). 
1) Vehicle Parameters

A vehicle size and mass must be chosen to calculate flight speed at loiter and the propeller thrust required to overcome drag at this speed. For this step, the characteristics of a monoplane Zimmerman planform were chosen. See Ref. 29 for the specific equations used in this step and for details about MAV planforms.

\section{2) Propeller Diameter}

Generally, propeller efficiency increases as a function of increasing diameter because the propeller forces act on a greater volume of fluid and blade stations that have poor efficiency are not overloaded. (In full-scale aircraft, there are other considerations, such as limited propeller tip speed to avoid compressibility effects, and limited diameter to ensure fuselage clearance). For a MAV it would be reasonable to choose a propeller diameter that was as large as possible in order to maximize efficiency while avoiding over-torquing the vehicle in the rolling axis. A ratio of the propeller diameter to the wingspan of 0.66 was chosen because it was observed on an existing operational MAV.

From steps 1) and 2), the following parameters were arrived at:

Table 1. Propeller design parameters

\begin{tabular}{lcll}
\hline \hline Diameter & 83.82 & $\mathrm{~mm}$ & $(3.3 \mathrm{in})$. \\
Loiter speed & 10.5 & $\mathrm{~m} / \mathrm{s}$ & $(23.5 \mathrm{mph})$ \\
Thrust & 7.33 & $\mathrm{~g}-\mathrm{f}$ & $(0.072 \mathrm{~N})$ \\
\hline \hline
\end{tabular}

With the propeller diameter, freestream velocity, and thrust as known variables, the rotational speed was taken as a free variable. The design space was explored using the QMIL minimum induced loss propeller code. A spinner diameter value of $12 \mathrm{~mm}$ was inputted into the program because it was known that the propeller would be tested with a motor of the same size. Any thrust created inboard of this area would therefore be likely to have been lost.

The design rotational speed was varied in increments of $100 \mathrm{rpm}$ from $J$ $=0.45$ to $J=1.9$. The trend of the design point efficiencies outputted from QMIL

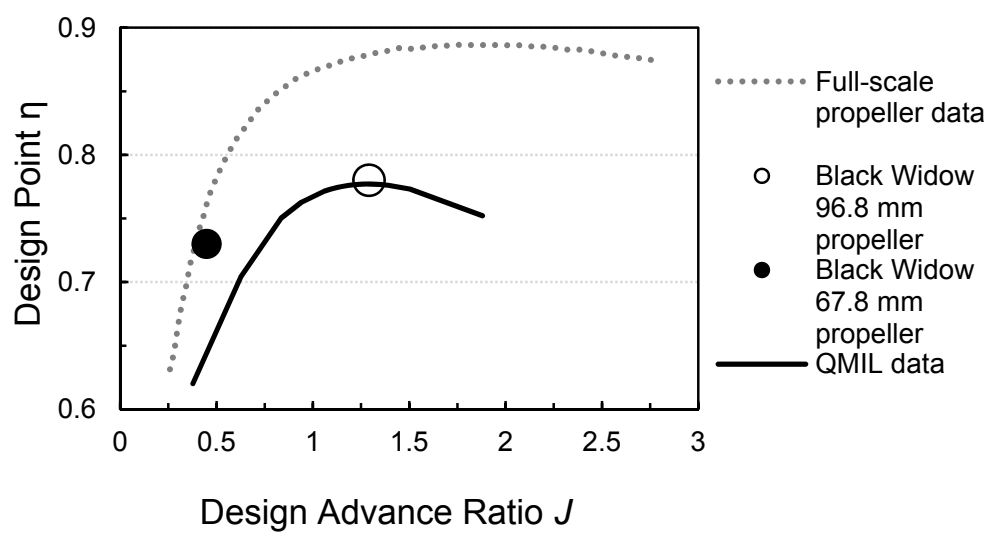

Figure 2. Peak efficiency vs. design advance ratio for full-scale propellers, ${ }^{30}$ the Black Widow MAV, ${ }^{4}$ and QMIL data.

is shown in Fig. 2. Data for full-scale propellers and the two Black Widow propellers were included for reference. It can be seen that the design point efficiencies predicted by QMIL are lower than those for full-scale propellers. This is due to the increased profile drag at low Reynolds numbers. The QMIL output showed that the peak design point efficiency increases until a design advance ratio of about $J=1.15$ (5700 rpm), stays nearly constant at about $77.6 \%$ efficiency through $J$ $=1.31(6500 \mathrm{rpm})$, and then begins to decrease more rapidly. Interestingly, the design point of the higher efficiency Black Widow propeller $(J=1.29)$ falls within this range of high efficiency. The lower efficiency Black Widow propeller did not match the trend as well.

To further narrow down the design rotational speed, off-design performance was taken into consideration. The propeller geometries outputted from QMIL for design speeds of 5900 - $6500 \mathrm{rpm}$ were inputted into QProp and the characteristic

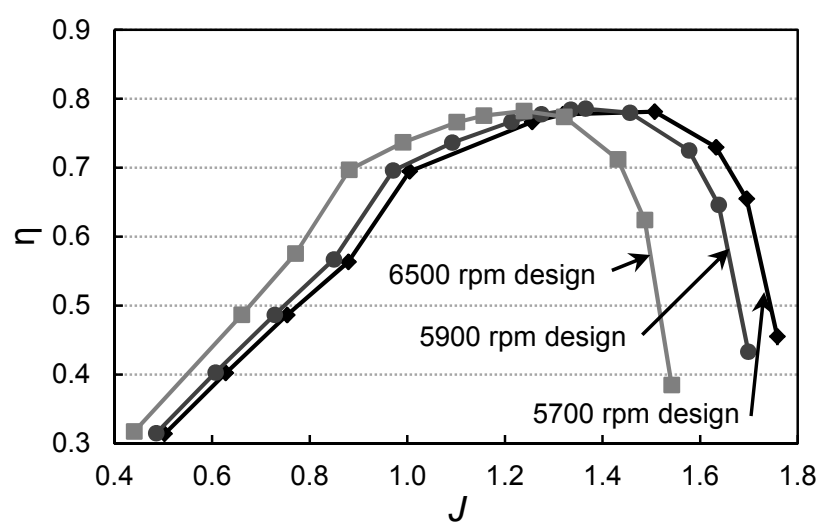

Figure 3. Propeller efficiency vs. advance ratio data from QProp for three QMIL designs.

American Institute of Aeronautics and Astronautics 
advance ratio curves were plotted. The plots of three of the designs (5700, 5900, and $6500 \mathrm{rpm})$ are shown in Fig. 3. It was observed that as the rotational speed was decreased from 6500 to $5900 \mathrm{rpm}$, the peak efficiency increased by about $0.1 \%$. This is due to reduced profile drag at higher blade Reynolds numbers at the slight expense of induced efficiency. As the design rpm decreases the range at which efficiencies of $70 \%$ or greater is reached also improves. However, blade chords also increase with decreasing design rpm so that the propeller can create the specified thrust. At a speed of $5700 \mathrm{rpm}$, the maximum blade chord approaches a length of one-half the outer radius, which is beginning to become impractical. Additionally, with such large chords this may approach the geometry at which lifting line theory loses applicability and other methods may be more appropriate, as suggested by Wald. ${ }^{26}$ The final design chosen was for a rotational speed of $5900 \mathrm{rpm}(J=1.27)$, which had a predicted peak efficiency of $77.7 \%$ and a geometric pitch-to-diameter ratio of 1.59 at $75 \%$ radius.

\section{Blade Airfoil Selection}

The next task was to refine the chosen airfoil section (Figs. 2 and 3 were made after the final airfoil sections were chosen and their properties inputted into QMIL and QProp). The QMIL output for the chord Reynolds number against radial position is shown in Fig. 4. It can be seen that Re ranges from about $5.0 \times 10^{3}$ to $2.1 \times 10^{4}$. Reynolds number is defined in Eq. (5). The Reynolds number is low at the root, where rotational speed and chords are small, as well as near the tips, where speed is high but MIL

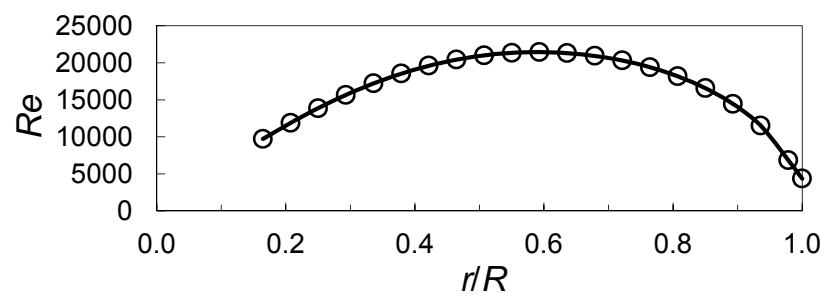

Figure 4. Blade chord Reynolds numbers of prototype propeller.

loading dictates small chords. In the range of Reynolds numbers of the propeller, airfoil choice is important because of reduced $L / D$ ratios and the problem of laminar bubble separation. The lift-curve slope is very sensitive to geometry and studies have shown that thin cambered airfoils with a sharp leading edge often have relatively high

$$
\operatorname{Re}=\rho \mathrm{cW} / \mu
$$

$L / D$ ratios when compared to airfoils with blunt or rounded leading edges. ${ }^{31}$ Airfoils are also found to be less sensitive to variation in Reynolds number and turbulence levels when compared to flat plates or a symmetrical 2sided airfoil such as the NACA 0012. Furthermore, it has been shown that the trailing edge geometry has less of an effect at low Reynolds numbers. ${ }^{31}$ For structural reasons, propeller airfoils often vary radially from a thin section at the tip to a thick section at the root. In this study it was decided that a uniform airfoil choice could be used for radii outside of the spinner since the large chords of the blades provided extra rigidity.

For the choice of airfoils, the first efforts were made to examine experimental low Reynolds number polars. The wind tunnel tests of Selig et al. ${ }^{32-34}$ at the University of Illinois at Urbana-Champaign (UIUC) produced force coefficients for many airfoil sections at Reynolds numbers of the $O 10^{4}-10^{5}$. These results were analyzed and the sections with the highest lift-to-drag ratios were chosen as candidates for the propeller airfoils. In particular, the data for the MA409 airfoil (Fig. 5) stood out. The MA409 is $6.69 \%$ thick at $23.8 \%$ chord with a maximum camber of $3.33 \%$ at $49.3 \%$ chord. Coordinates for the airfoil can be found in Ref. 33. At a Reynolds number of $4.1 \times 10^{4}$ the MA409 showed a remarkably smooth polar and did not exhibit significant effects of separation. Selig et al. measured a large $L / D$ of 35 at a reasonable angle of attack of 4.3 degrees. This was therefore chosen as the base airfoil profile.

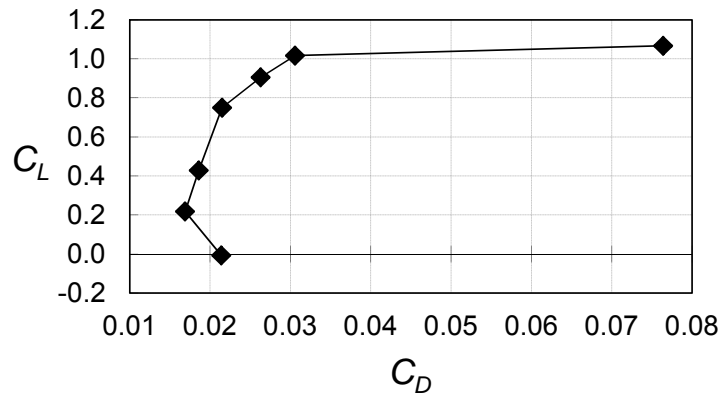

Figure 5. UIUC experimental data for MA409 airfoil at $R e=4.1 \times 10^{4}$.

\section{Airfoil Modifications}

It is well known that at a Reynolds numbers of $O 10^{4}$ thin airfoils have benefits over thicker sections. ${ }^{35,36}$ A study by $\mathrm{Kunz}^{37}$ showed that at a Reynolds number of $6.0 \times 10^{3}$, decreasing NACA 4-digit sections from $8 \%$ to $2 \%$ increased both the maximum $L / D$ ratios as well as the maximum lift coefficients. These observations led the authors to reduce the thickness of the MA409 airfoil while maintaining its general profile. The maximum camber, location of maximum camber, and location of maximum thickness remained unchanged. It was decided that a maximum thickness of $4 \%$ was the thinnest possible for practical manufacturing and sufficient blade stiffness. Lastly, the 
trailing edge thickness was increased from $0.07 \%$ to $0.5 \%$ to facilitate fabrication. The profiles of the original and the modified 4\% thick MA409 airfoils are shown in Fig. 6.

XFOIL $^{18}$ v. 6.94

was used to obtain

force coefficients for

the modified airfoil.

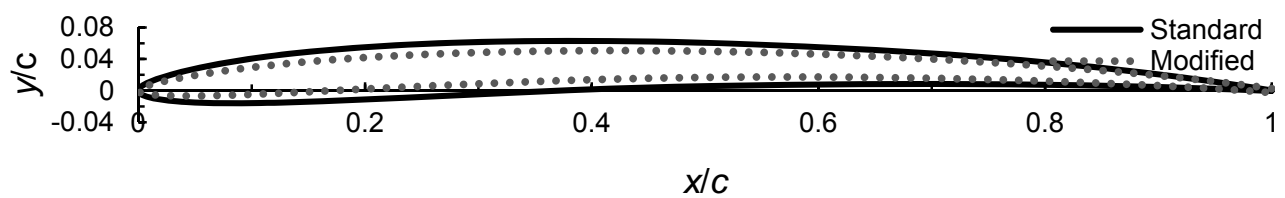

One of the userinputs in XFOIL is the $N$ value for the

Figure 6. Standard (6.69\% thick) and modified (4\% thick) MA409 airfoil.

$e^{N}$ method transition criteria. Lian and Shyy ${ }^{38}$ found that a value of $N=8$ gave numerical results that were in good agreement with experimental results at $R e=6 \times 10^{4}$ for a low Reynolds number airfoil. In the Reynolds number range of the propeller design $\left(5.0 \times 10^{3}-2.2 \times 10^{4}\right)$, the force coefficients from XFOIL for the $4 \%$ thick MA409 airfoil were found to be insensitive to the $N$ value when it was set between 7 and 9 (the standard value). The value $N=7$ was ultimately chosen because it gave the best agreement with the experimental data for the standard MA409 airfoil (Fig. 5). With the settings chosen, XFOIL was run at Reynolds numbers from $1.0 \times 10^{4}$ to $2.2 \times 10^{4}$. As expected, the results showed reduced drag and increased maximum lift coefficients compared to the standard airfoil. From the polars, the design airfoil coefficient of lift was chosen to be a constant 0.5 . This value was chosen as a compromise between high lift at the design point and a safety margin in angle of attack before stall at off-design conditions.

\section{Airfoil Lift and Drag Prediction in QProp}

Both QProp and QMIL require the user to input the airfoil characteristics, including lift slope, the minimum drag coefficient, as a 2-piece parabolic curve fit to the data (for details, see Ref. 22). The data can be obtained experimentally or numerically. The programs take these inputs and extrapolate the drag coefficient as a function of $C_{l}$ and $R e$. The relation used in the non-stalled region is given in Eq. (6),

$$
C_{D}\left(C_{L}, R e\right)=\left[C_{D 0}+C_{D 2} \times\left(C_{L}-C_{L C D O}\right)^{2}\right] \times\left[R e / R e_{r e f}\right]^{R e x p}
$$

where $C_{D 2}$ controls the slope of the parabola and changes above and below the coefficient of lift at which drag is a minimum before negative stall. In this study there were no experimental results for the airfoil so the XFOIL data was used. A comparison of the XFOIL results and the curve fit is given in Fig. 7. This parabola does not take into account the characteristic "kink" from separation, but offers a good fit for the XFOIL results for the thinned airfoil

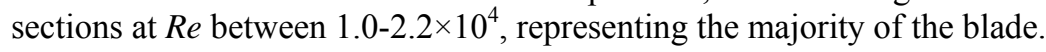

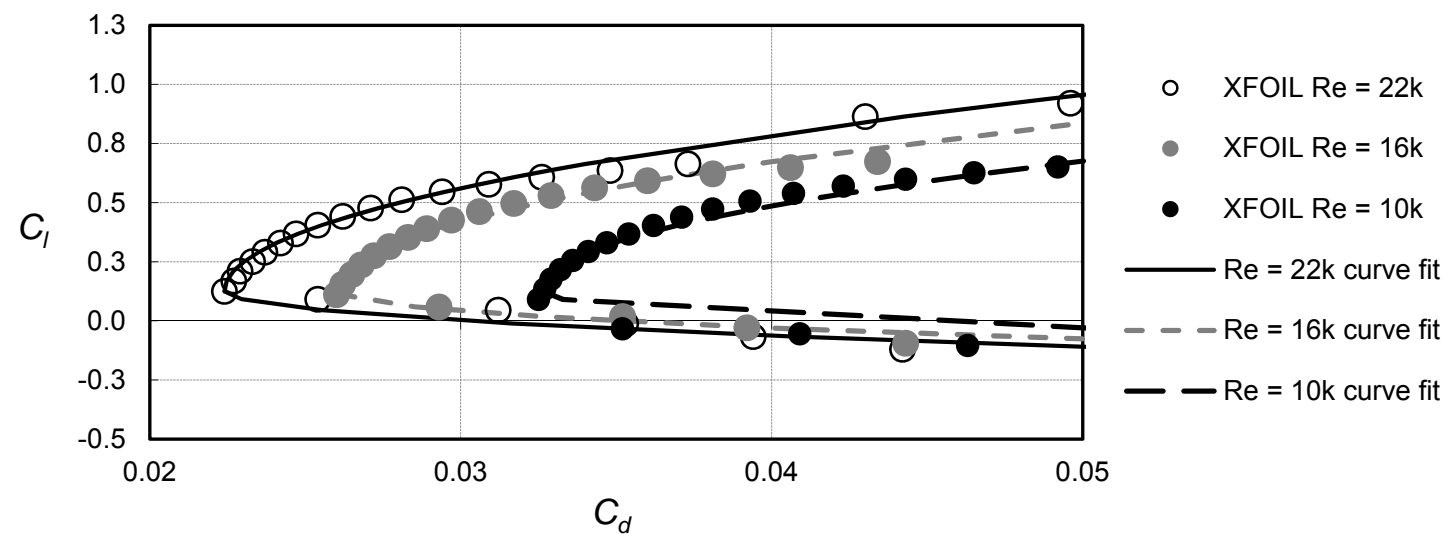

Figure 7. XFOIL polars of modified MA409 with $4 \%$ maximum thickness and $0.5 \%$ thick trailing edge and QMIL/QProp curve fits. 
It is difficult to assess the accuracy of the airfoil data from XFOIL. For example, the data shows what appears to be an optimistic lift-to-drag ratio $(L / D)$ greater than 17 at $C_{L}=0.5$ and $R e=2.2 \times 10^{4}$. Some have suggested applying corrections to XFOIL data at low Reynolds numbers. For example, in their propeller models, Uhlig and Selig ${ }^{39}$ reduced the maximum lift coefficient predicted by XFOIL by $10 \%$, based on "experience with XFOIL." Although there exist no experimental results for the modified MA409 geometry for comparison, experimental results for other airfoil sections can be used as rough reference points. For example, Selig et al. ${ }^{32}$ measured a $L / D_{\max }$ of about 35 for a standard $6.69 \%$ thick MA409 at $R e=4.1 \times 10^{4}$. Laitone $^{31}$ measured a $L / D_{\max }$ of

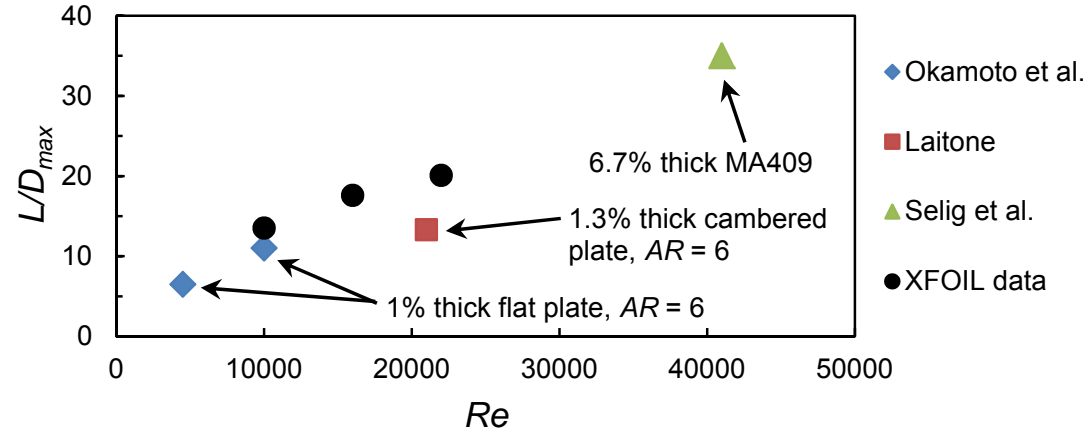

Figure 8. XFOIL predictions of $L / D_{\max }$ of modified MA409 compared to experimental results for various airfoils.

13.3 for a $1.3 \%$ thick, $5 \%$ cambered plate section at $R e=2.1 \times 10^{4}$. Hansen and Bowman ${ }^{40}$ measured a $L / D_{\max }$ of just 7 for a $3 \%$ thick, $6 \%$ cambered plate at $R e=2 \times 10^{4}$. Okamoto et al. ${ }^{36}$ measured a $L / D_{\max }$ of 11.0 for a $3 \%$ thick, $5 \%$ cambered plate at $R e=1.0 \times 10^{4}$. Kunz ${ }^{37}$ predicted an $L / D_{\max }$ of about 13 for his optimized $2 \%$ thick airfoil at $R e=$ $6.0 \times 10^{3}$, and an $L / D_{\max }$ as high as 15.7 for $2 \%$ thick NACA 4-digit airfoils at $R e=1.2 \times 10^{4}$. Fig. 8 shows the XFOIL data plotted alongside experimental results for a selection of airfoils. It can be seen that the predicted $L / D$ ratios in XFOIL for the 4\% thick airfoil appear high, but not unreasonably so. The final propeller geometry is shown in Fig. 9. The chord and blade distributions begin at a radial station just beyond $6 \mathrm{~mm}$ because, as mentioned earlier, the propeller was designed for use with a $12 \mathrm{~mm}$ diameter motor.

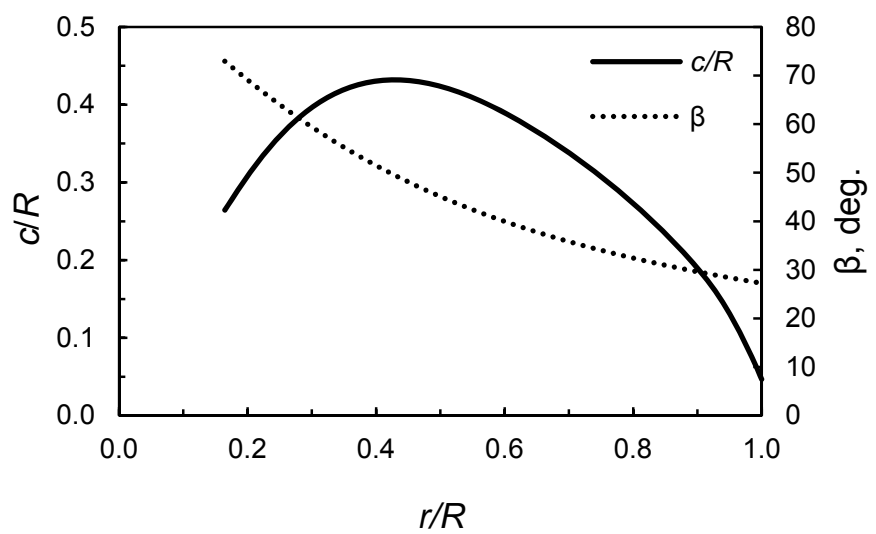

Figure 9. Prototype propeller chord and twist distribution.

\section{Propeller Fabrication}

The small dimensions and highly three-dimensional geometry of MAV propellers make fabrication a challenge. At the same time, accuracy in the propeller geometry is critical to performance. For example, an error of one degree in blade angle can result in large variances in section lift coefficient, possibly inducing stall, and the error can propagate further outboard along the blade. To select a method of fabrication, available options were examined according to their individual pros and cons and feasibility in the scope of the study.

\section{Rapid Prototyping}

The authors are unaware of any operational MAV propellers that were made by using rapid prototyping, except in the creation of molds. ${ }^{4,41}$ However, rapid prototyping technologies such as stereolithography and PolyJet show promise in building the propeller itself. In particular, PolyJet technology has the capability to produce parts with layer thicknesses of just $16 \mu \mathrm{m}$. It was unknown, however, whether the mechanical properties of the PolyJet material would be sufficient to withstand the inertial and aerodynamic loads of the propellers. In this study, a PolyJet 
propeller was made to test the capabilities of the technology. The prototype is shown in Fig. 10. It was found that while the process could create blades as thin as $51 \mu \mathrm{m}$ on the leading and trailing edges, the twist of the blades was deformed. This perhaps occurred during removal of the support material. Because the chord and thickness distributions were accurate, this propeller could have potentially been re-pitched to its correct twist on a fixture and bonded to a stiff material such as carbon fiber fabric. However, the advantage of rapid prototyping in the first place is that molds are not required, so the authors decided to pursue other fabrication options.

\section{Electro-Discharge Machining (EDM) and Laser Micromachining}

EDM was initially considered to be an excellent option due to its high resolution and the low forces exerted on the propeller during fabrication. However it was eliminated because custom electrodes would be required to cut the concave portions of the propeller blades.

Laser micromachining was considered a promising and novel option worth attempting in the future. This process can be used to cut many metals and ceramics that cannot be conventionally machined, and with great precision.

\section{Injection Molding}

Injection molding of plastics was considered a proven technology because it is used in the hobby industry to make propellers of MAV-size. Injection molding can create very thin, accurate geometries and is also a relatively fast process. This was a good option but was eliminated because the authors did not have sufficient experience designing molds and because of the risk that the plastic would not flow into the thin propeller blade cavities.

\section{Computer Numerical Control (CNC) Machining}

Another option was to fabricate the propellers using conventional CNC machining. MAV-size propellers can be machined from metals, plastics, or woods and high tolerances are possible. Details of this process can be found in Refs. 42 and 43 . The challenges of this process lie in supporting and aligning the blade after the first surface has been cut. If not done correctly, the thin blades may not survive the machining cutting forces. An approach used by Cheng ${ }^{42}$ was to machine the first side of the propeller, and then flip it onto a support fixture designed to mate with the cut surface. This fixture was attached to vacuum hoses which held the propeller in place with suction. While $\mathrm{CNC}$ machining is a proven manufacturing technique, it was eliminated because of the extra time required to manufacture the 3-D fixtures and the great precision required for alignment.

\section{Composites Fabrication}

A composite lay-up was the option finally selected for propeller fabrication. Composites offer extremely high stiffness and the molds can be re-used many times. Composites are a relatively proven method; they have been used to make MAV and UAV-size propellers as well as a small selection of commercial MAV-size propellers. It was decided that two-piece molds would be used so that both the upper and lower surface of the propeller airfoils could be governed.

\section{Molds for Composites Fabrication}

The two-piece mold designs were created in Siemens NX modeling software. The airfoil blade angle and chord outputs from QMIL were imported, and the modified airfoils were positioned along an axis about their aerodynamic centers. For stiffness, NACA 4-digit airfoils with greater than $20 \%$ thickness were chosen for the root of the propeller between the hub and the first blade section. The airfoils were then surfaced and the molds were created by extruding the blade surfaces to create a solid geometry. The molds were designed for a wet layup process since the mold could not survive the temperatures in an autoclave during the curing of prepreg composites. The final molds, which were made with using PolyJet rapid prototyping, are shown in Fig. 11. The molds have several important features, including a space for excess woven fabric, epoxy and air to escape at the trailing edges, cutouts to facilitate separation of the mold halves, and a cylinder in the hub that creates a hole for a press fit onto the motor shaft. Allowing space for the fabric to escape the mold cavity is done to allow some leeway in the shape of the fabric blade templates and to

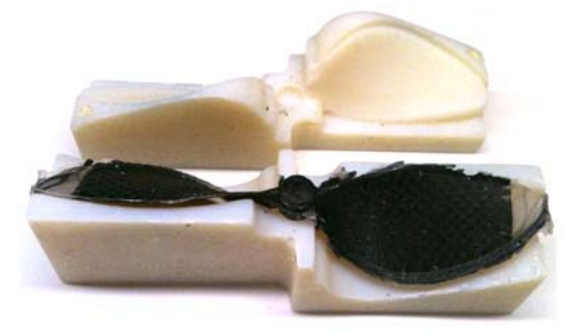

Figure 11. Molds with cured propeller. 
ensure that the trailing edge is carbon and not only epoxy, which could easily flake away. This feature also means that during trimming the trailing edge must be lightly sanded down to its final intended thickness.

\section{Propeller Composites Fabrication}

Epon 862, an epoxy which cures at room temperature, was chosen for the composites fabrication. Each blade was made from two pieces of fabric. The blade was primarily composed of a piece of $0.14 \mathrm{~mm}$ thick plain-weave carbon fiber with a tow count of $1 \mathrm{k}$. The outer $15 \%$ of each blade was made from a piece of $0.05 \mathrm{~mm}$ plain-weave fiberglass which slightly overlapped the carbon. The fabric pieces were cut using templates, coated with epoxy, and aligned in the mold. The hub was then filled with a mass of chopped carbon fibers and epoxy. Once the molds were pressed together the epoxy filled the cavities and excess material was forced out.

\section{Trimming and Balancing}

After curing, the blade profiles required trimming to remove excess material. Computer-controlled cutting methods such as $\mathrm{CNC}$ machining and waterjet cutting were initially considered, but the cutting forces from these processes necessitates the use of a sacrificial fixture to support the blade, adding complexity to the process. An attempt was made to use a continuous wave laser to cut a sample of carbon fiber plate, but results were unsatisfactory through a range of power levels and cutting speeds. It was found that the parting lines from the molds provided a visual reference for trimming by hand, and the propellers were ultimately trimmed using a rotary hand tool and various grades of sandpaper. Next the propeller was balanced by filing material off of the hub, where aerodynamic performance is least affected. The final trimmed and balanced propeller is shown in Fig. 12. The propeller appeared to be symmetric when spun at high speed and viewed from the side. A rigorous validation of the geometry was not conducted, but measurement with digital calipers revealed that at the radius of maximum width the chord was about $0.4 \mathrm{~mm}$ less than as designed. A brief analysis using QProp showed that removing a constant $0.4 \mathrm{~mm}$ from the blade profile design reduced thrust by about $0.17 \mathrm{~g}-\mathrm{f}(-2.4 \%)$, and torque by $0.039 \mathrm{mNm}(-2.5 \%)$ at the design point. The loss in thrust was in the range of the uncertainty of the thrust measurement, which was considered acceptable. Similarly, the loss in torque was about the same percentage, which indicates that the efficiency of the propeller should not change considerably at the design point. It was assumed that the blade thickness distribution was correctly defined by virtue of the two-sided molds fitting when pressed together.

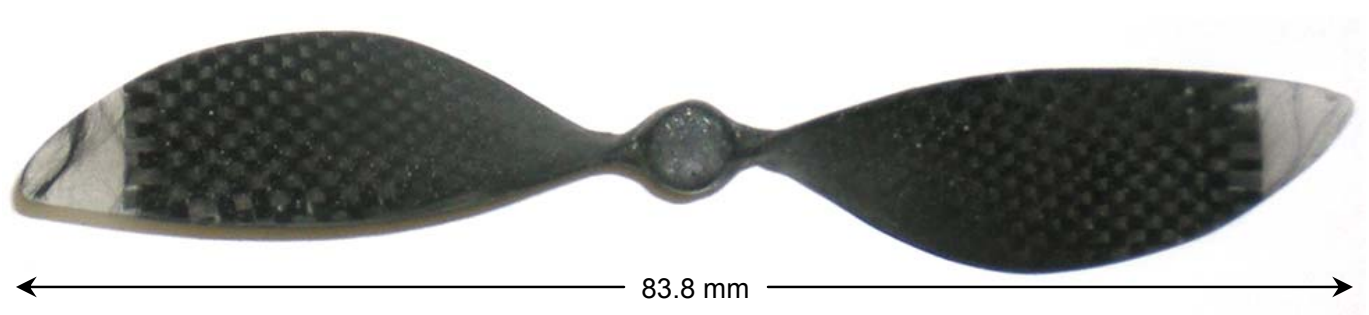

a)

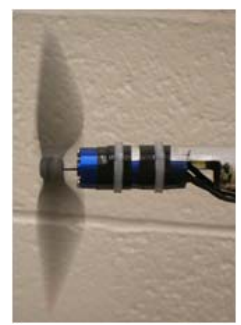

b)

Figure 12. a) Trimmed and balanced prototype propeller. b) side-view of propeller while spinning.

\section{Test Stand Design}

One of the challenges of micro propeller and rotor testing is the measurement of the small thrust forces and torques. Reference 44 describes some of the challenges in designing test stands for small rotors. To adequately compare the performance of the prototype propeller to the analytical model, the goals of the test stand were to have a minimum resolution of 0.5 grams-force (g-f) of thrust and $100 \mu \mathrm{Nm}$ of torque, and to minimally disturb the propeller wake. Thrust is relatively easy to measure, but reliably measuring the propeller torques can be difficult. There were three practical options for measuring the torque: (i) Motor manufacturer specifications can be used. Modern coreless and brushless motors have constants given by manufacturers such as no-load speed, voltage (or speed) constant, and no-load torque. These constants can be used to construct a characteristic curve of the motor. However, these constants may vary by more than $\pm 10 \%$ from one motor to the next, which was not acceptable in this study. (ii) A dynamometer can be used to characterize the motor. A dynamometer applies a range of known resistive torques at different voltages and speeds and provides data on current draw. An advantage to using a dynamometer is that there are commercially available dynamometers in the size range of MAV motors. The drawbacks to using a dynamometer are that a large amount of data must be taken and the performance of small motors fluctuates with temperature and degrades over time. ${ }^{45}$ These complications add uncertainty to the 
measurements so dynamometer testing was rejected. (iii) The test stand can simultaneously measure thrust and torque. This option is the simplest and has the least uncertainty, but if coupling exists between the force measurements it must be characterized and be predictable. This option was ultimately chosen.

\section{First Test Stand}

To measure the performance of the prototype propeller, a test stand was designed and constructed. The first design, shown in Fig. 13, used two beam load cells obtained from commercially available hand-held scales. Grasmeyer and Keennon ${ }^{4}$ reported using load cells from lab scales, although their stand used three load cells in a different configuration. The thrust measurement is made by a load cell which connects directly to the motor and is bolted to an aluminum bar. This bar pivots at the bottom on an axle running through two ball bearings. The torque measurement is made by the second load cell which rests against the bar at a very small moment arm so that torque is magnified. This bar is maintained at an angle slightly off of vertical so that it always rests against the torque load cell even if the propeller is not spinning. The goal of this design was to measure torque at a distance from the propeller, without a bulky mechanism near the propeller to reduce the disturbance to the propeller wake. Unfortunately, static propeller tests showed that the torque measurement was not repeatable. Several different beam load cells with resolutions ranging from $0.01 \mathrm{~g}-\mathrm{f}$ to $0.05 \mathrm{~g}$-f were tried, but repeatable results could still not be obtained, perhaps due to hysteresis in the pivoting mechanism. It was also found that torque was non-linearly coupled with thrust. Because of these inherent problems, development of the first test stand design was halted.

\section{Second Test Stand}

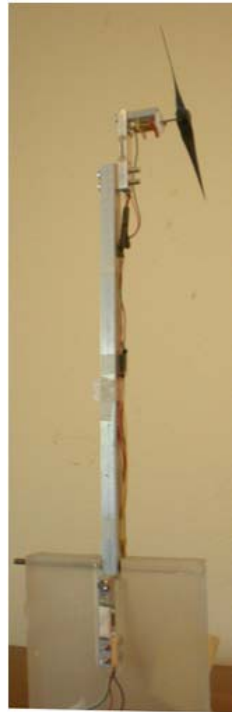

Figure 13. First test stand.

A second test stand was developed to use the ATI Nano 17 6-axis load cell, which has listed resolutions of 0.3 gf thrust and $16 \mu \mathrm{Nm}$ torque. The load cell was mounted to a fairing and attached with an adaptor to a hollow aluminum tube which in turn held the motor. The motor and load cell were separated by a distance to reduce heat transfer and blockage of the propeller wake. The motor chosen was the brushless Medusa MR-012-030-4000, which has a diameter of $12 \mathrm{~mm}$. The motor was controlled by a Castle Creations Thunderbird-9 electronic speed controller (ESC). To minimize hysteresis, flexible wires arranged in a large loop were used to bridge the ESC and the fairing. The second test stand is shown in Fig. 14.

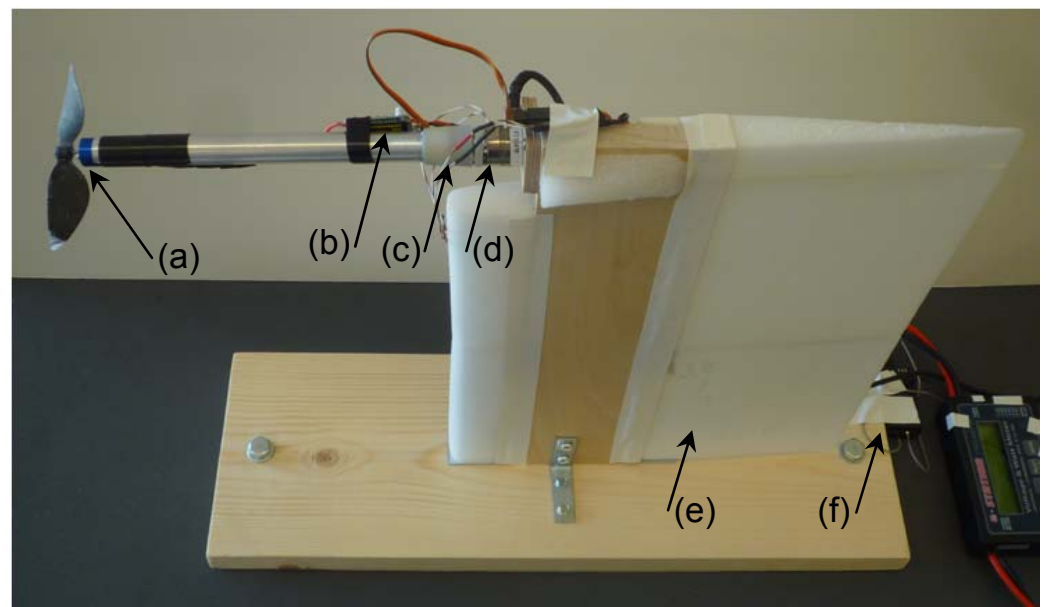

Figure 14. Second test stand. (a) motor; (b) ESC; (c) adaptor; (d) load cell; (e) fairing; (f) receiver.

\section{Validation of Thrust Calibration}

To verify the factory calibration of the load cell, the thrust measurement was validated. This was done by placing known masses on the load cell when the thrust axis was oriented vertically. The readings showed perfect agreement with the calibration and the bias stayed constant after the transducer was loaded and unloaded repeatedly. 


\section{Validation of Dynamic Measurements with a Commercially Available Propeller}

To verify the accuracy of the second test stand during dynamic operation with a spinning motor and propeller, the static thrust and torque of an $80 \mathrm{~mm}$ Union U-80 propeller were measured. Data was collected over a period of 5 seconds at a rate of $500 \mathrm{~Hz}$. The results were compared with independent experiments for thrust and torque by Deters and Selig ${ }^{46}$ and thrust by Chapman, Liu, and Moschetta ${ }^{47}$ on the same propeller. The reference datasets cover different speed ranges but intersect between 7,500 and 10,000 rpm. In this overlapping region, the two data sets agree very well on the thrust of the U-80. The combined datasets are shown in Fig. 15.

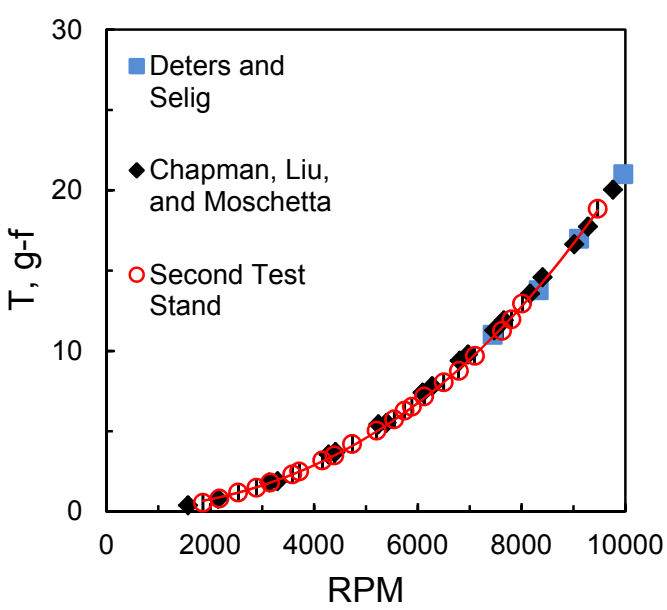

a) Static U-80 thrust

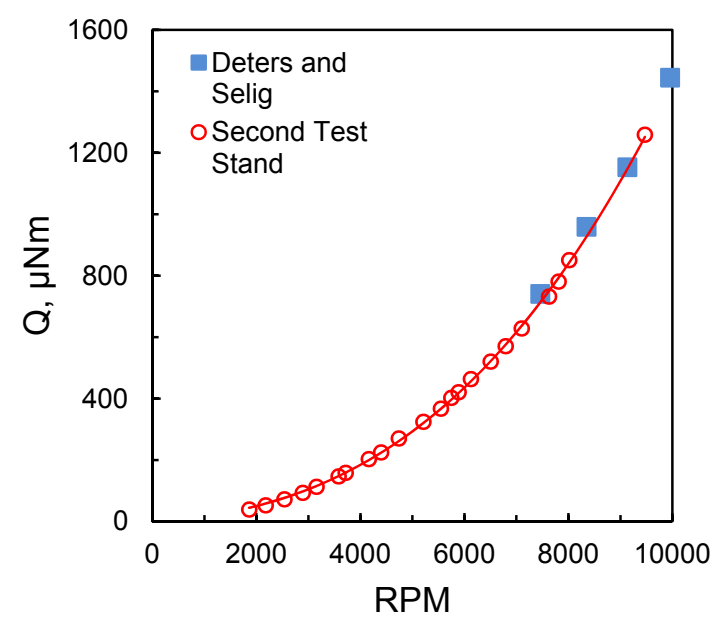

b) Static U-80 torque

Figure 15. Measured thrust and torque of the U-80 propeller.

The thrust measurements from the second test stand showed excellent agreement with the measurements from both datasets. The torque measurements were smooth and appeared to very closely follow the trend of the data of Deters and Selig (who coincidentally used the same model motor as in the present study). The uncertainty in the data collected with the second test stand was calculated by finding the $95^{\text {th }}$ percentile confidence interval. In both the thrust and torque plots the uncertainties were too small to see. These results were considered ample validation of the test stand during dynamic testing.

\section{Dynamic Testing of Prototype Propeller}

After completion of the calibration of the second test stand, the prototype propeller was tested in the $2 \mathrm{ft} . \times 2 \mathrm{ft}$. open-circuit subsonic wind tunnel at the University of Michigan, which has a contraction ratio of 17:1. The test stand was placed at the bottom of the wind tunnel and bolted in place. The propeller was tested at the design point and also at different freestream velocities to find off-design performance.

A 16 bit $\mathrm{ADC}$ that was capable of taking raw strain gauge measurements at a sampling frequency of up to $3 \mathrm{kHz}$ was used for force measurements. Each data point was actually collected over a period of 5 seconds at a rate of 1 $\mathrm{kHz}$. For more details on the embedded software and DAQ configuration, see Ref. 48. Propeller rotational velocity was measured using an infrared LED pulsed at $38 \mathrm{kHz}$ mounted on the test stand, paired with a photodetector mounted on the inside of the wind tunnel. This data was collected by a data acquisition unit made by Iosix and sent to a PC. A strobe was also used to verify the rpm measurements. Freestream velocities were calculated from Pitot probe measurements and verified using a digital ultrasonic anemometer. We did not take data at freestream velocities less than $1 \mathrm{~m} / \mathrm{s}$ below the design velocity because our motor, which did not have a gearbox, was unable to do so without overheating. Measurements were taken without a spinner because the commercial rubber spinner used was difficult to balance at high speeds, resulting in poor data. Lastly, thrust measurements conducted during wind tunnel testing showed higher uncertainties than those conducted in static conditions. This may be due to unsteady phenomena or vibrations related to the freestream flow. However, the uncertainty was considered low enough that qualitative and quantitative conclusions could be drawn. 


\section{Results and Discussion}

Plots of the thrust, torque, and efficiency of the prototype propeller at freestream speeds of $10.5 \mathrm{~m} / \mathrm{s}$ and $12.5 \mathrm{~m} / \mathrm{s}$ are shown in Fig. 16. It can be seen that the results generally follow the trends of the QProp predictions, but that both thrust and torque measurements are lower than predicted. Efficiencies were about $20 \%$ below prediction. Two logical explanations for the disagreement between the experiment and prediction might be (i) uncertainties in the aerodynamic inputs in the vortex theory code and (ii) manufacturing defects.

In regard to the aerodynamics, a considerable source of error may be due to the airfoil force coefficients. At the UAV-scale, Moffitt et al. found these inputs to be the largest source of uncertainty in their prediction models. ${ }^{21}$ This source uncertainty would only increase with reduced Reynolds numbers. The thrust measurements at both freestream speeds show are lower than prediction, with agreement slightly better at low rotational speed. This discrepancy in thrust, as well as the fact that the efficiency curves are offset from the QProp predictions may indicate that the desired lift coefficients were not being reached at the designed blade angles.

The torque measurements results agree well with QProp predictions at low rotational speeds, but agreement worsens with increasing rotational speed. The $10.5 \mathrm{~m} / \mathrm{s}$ and the $12.5 \mathrm{~m} / \mathrm{s}$ torque data both show this trend. This may indicate that the slope of lift vs. drag is actually steeper than modeled, or that positive stall occurs earlier than the XFOIL polars suggest.

As mentioned previously, another issue is that at the design advance ratio of $J=1.28(\lambda=0.41)$ of the prototype propeller, the circulation distribution using the $F$ factor deviates significantly from that using the Goldstein factor, as shown in Fig. 1. This deviation may have changed the loading far enough from the Betz condition to increase the induced losses. How sensitive the geometry is to the radial momentum loss factor and how sensitive the efficiency is to the geometry has not been studied thoroughly and merits further investigation.

Regarding the influence of inaccuracies in the geometry of the fabricated propeller, in this study only one sample of the propeller prototype design was tested. The sample tested had chords slightly smaller than the design geometry, but reduction in thrust lies within the uncertainty range of the thrust measurement, and the predicted reduction in torque was likewise very small. This suggests that errors that occurred during trimming are probably not largely to blame for the reduced performance. It must be mentioned that other sources of error in the geometry are possible. For example, errors in the blade thickness distribution were not quantified in this study but may have existed. Cheng ${ }^{42}$ and $\mathrm{Kunz}^{37}$ used laser scanning and measured angle static deformations as great as 6 degrees in their $25 \mathrm{~mm}$ diameter epoxy rotors, and greater than 2 degrees in aluminum rotors of the same dimensions. It was undetermined whether these deformations came from handling or during a step in the manufacturing process.

Further deformations may occur during spinning from dynamic inertial and aerodynamic loads. During static testing of the U-80 propeller, significant deflections in the thrust axis were observed. A task for future work is to predict propeller deflections and to compare them to measured deflections.

A final potential explanation is that the QMIL and QProp vortex theory methodologies are not suitable at Reynolds numbers this low. However, the work of Youngren and Chang ${ }^{19}$ showed good agreement between the predictions of XROTOR (which has the same theoretical formulation as QProp and QMIL but with some additional design options) and a laminar 3-D numerical simulation at Reynolds numbers close to those of the present study. Also, Grasmeyer and Keennon ${ }^{4}$ showed that a similar minimum induced loss methodology could provide very good agreement with experimental data for a MAV propeller. Therefore it is expected that a vortex/blade element theory will offer guidance for MAV propeller design at low Reynolds numbers.

From a design point of view, although the peak efficiencies of $60 \%$ were lower than predicted, the results were somewhat encouraging when it is considered that many hobby propellers greater than $22 \mathrm{~cm}$ in diameter that operate at significantly higher Reynolds numbers do not achieve $60 \%$ efficiency. ${ }^{16}$ (This may be due in part to the low-pitch design of most hobby propellers that provides greater thrust at low speeds but compromises peak efficiency). The U80 propeller that is commonly used on MAVs was found to reach a peak efficiency of just $31 \%$ in a freestream. ${ }^{5}$ 


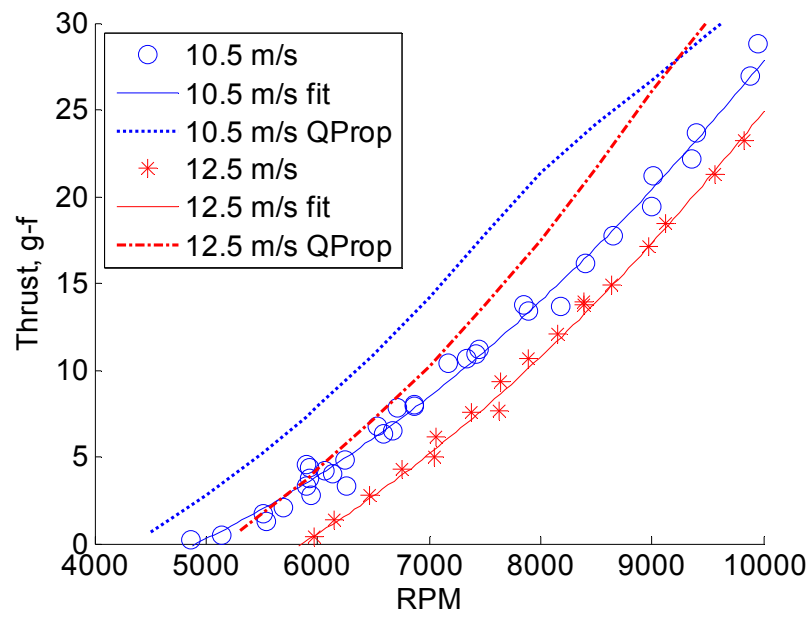

a) Thrust

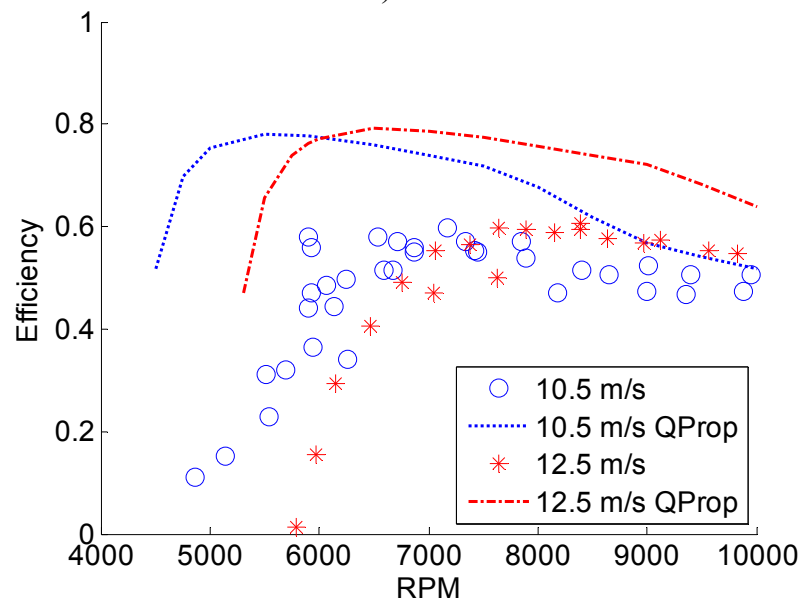

c) Efficiency

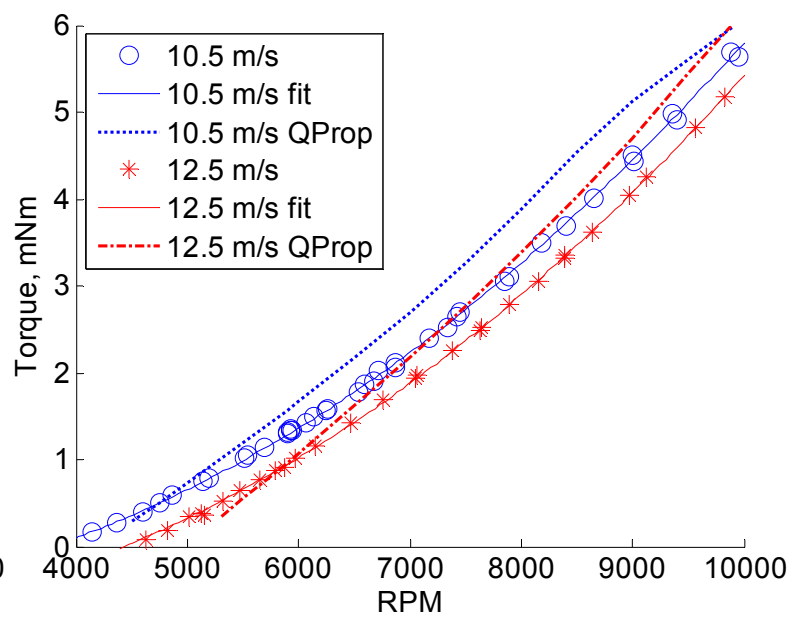

b) Torque

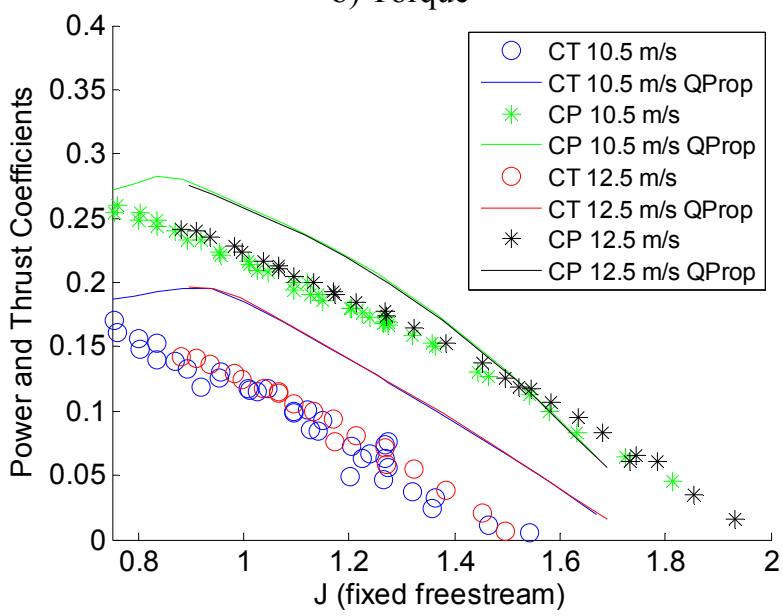

d) Coefficients

Figure 16. Prototype propeller thrust, torque, efficiency, and coefficients at $10.5 \mathrm{~m} / \mathrm{s}$ and $12.5 \mathrm{~m} / \mathrm{s}$ and QProp predictions.

\section{Summary and Conclusions}

This article describes the construction and validation of a test stand suitable for MAV-size propellers and a fabrication process using molded carbon fiber composites. The propellers were stiff, durable, and thin. However, there was room for improvement in the trimming process, which may have introduced uncertainty in the geometry of the prototype. A propeller was designed using the minimum induced loss vortex theory of QMIL to the parameters of a MAV. Wind tunnel results showed lower thrusts and torques than predicted by QProp. Efficiencies were about $20 \%$ lower than predicted. The results add to the literature and suggest that at the MAV scale, vortex theory with 2-D airfoil data inputs can provide at the least a good preliminary design tool. The discrepancies between experiment and analysis may be due, in part, to uncertainties in the airfoil force coefficients obtained from XFOIL. It is possible that corrections could be applied to the 2-D airfoil data to improve correlation. Another potential explanation for the variation between results and predictions includes the possibility that the Prandtl momentum loss factor is not ideally suited for use with a high advance ratio, two-bladed MAV propeller. Lastly, fabrication error may have played a part in the discrepancies. The fabrication process and experimental apparatus described in this article may facilitate the future investigation of these issues.

\section{Acknowledgments}

The authors thank Professors Ella Atkins, Luis Bernal, Peretz Friedmann and Jyoti Mazumder for advice and assistance. We also thank Mr. Devesh Kumar and Mr. Jack Norris for sharing their technical expertise. 


\section{References}

${ }^{1}$ McMichael, J. M., and Francis, M. S., "Micro Air Vehicles - Toward a New Dimension in Flight," DARPA, 1997.

${ }^{2}$ Mueller, T. J. and DeLaurier J. D., "An Overview of Micro Air Vehicle Aerodynamics," Fixed and Flapping Wing

Aerodynamics for Micro Air Vehicle Applications, Vol. 195, Progress in Astronautics and Aeronautics, AIAA, 2001, pp. 1-10.

${ }^{3}$ Shyy, W., Berg, M., and Ljungqvist, D., "Flapping and Flexible Wings for Biological and Micro Air Vehicles," Progress in Aerospace Sciences, Vol. 35, 1999, pp. 155-205. 2001 .

${ }^{4}$ Grasmeyer, J. M., and Keennon, M. T., "Development of the Black Widow Micro Air Vehicle," AIAA 2001-0127, Jan.

${ }^{5}$ Peterson, B., Erath, B., Henry, K., Lyon, M., Walker, B., Powell, N., Fowkes, K., and Bowman, W. J., "Development of a Micro Air Vehicle for Maximum Endurance and Minimum Size,” AIAA 2003-416, Jan. 2003, pp. 3-4.

${ }^{6}$ Cho, L., Yoon, J., Cheolheui, H., and Cho, J., "Aerodynamic Design and Analysis of a Propeller for a Micro Air Vehicle," Journal of Mechanical Science and Technology, Vol. 20, No. 10, pp. 1753-1764, 2006.

${ }^{7}$ Larrabee, E. E., "Five Years Experience with Minimum Induced Loss Propellers. I - Theory," Society of Automotive Engineers Paper No. 840026, International Congress and Exposition, Detroit, Feb. 1984.

${ }^{8}$ Rankine, W. J. M., Transactions, Institute of Naval Architects, Vol. 6, 1865, p. 13.

${ }^{9}$ Froude, W., "On the Elementary Relation between Pitch, Slip and Propulsive Efficiency," Transactions of the Institute of Naval Architects, Vol. 19, 1878, pp. 47-57.

${ }^{10}$ Betz, A., "Schraubenpropeller mit geringstem Energieverlust," Göttinger Nachrichten, 1919, p. 192-213.

${ }^{11}$ Prandtl, L., Ibid.

${ }^{12}$ Goldstein, S., "On the Vortex Theory of Screw Propellers," Proceedings of the Royal Society of London, Series A, Vol. 123, No. 792, 1929, pp. 440-465.

${ }^{13}$ Theodorsen, T., Theory of Propellers, New York: McGraw-Hill Publications in Aeronautical Science, 1948.

${ }^{14}$ McCormick, B. W., Aerodynamics, Aeronautics and Flight Mechanics, John Wiley \& Sons, 1994, p. 304.

${ }^{15}$ Adkins, C. N., and Liebeck, R. N., "Design of Optimum Propellers," AIAA 83-0190, Jan. 1983.

${ }^{16}$ Brandt, J. B. and Selig, M. S., "Small-Scale Propeller Performance at Low Speeds," Master's Thesis, University of Illinois at Urbana-Champaign, April 2005.

${ }^{17}$ Lee, K. H., Jeon, Y. H., Bae, E. S., and Lee D. H., "Implementation of the Numerical Optimization for the Micro-Air Vehicle Propeller," AIAA 2004-4428, August 2004, pp. 2-3.

${ }^{18}$ Drela, M., XFOIL: An Analysis and Design System for Low Reynolds Number Airfoils. Low Reynolds Number Aerodynamics, Springer-Verlag, New York, 1989, pp. 1-12.

${ }^{19}$ Youngren, H., and Chang, M., "Test, Analysis and Design of Propeller Propulsion Systems for MAVs," AIAA 2011-876, Jan. 2011.

${ }^{20}$ Drela, M., and Youngren, H., XROTOR, Massachusetts Institute of Technology, Cambridge, MA, http://web.mit.edu/drela/Public/web/xrotor/, 2011.

${ }^{21}$ Moffitt, B. A., Bradley, T. H., Parekh, D. E., and Mavris, D., "Validation of Vortex Propeller Theory for UAV Design with Uncertainty Analysis," AIAA 2008-406, Jan. 2008.

${ }^{22}$ Drela, M., QProp Propeller/Windmill Analysis and Design, Ver. 1.22, Massachusetts Institute of Technology, Cambridge,

MA, http://web.mit.edu/drela/Public/web/qprop/, 2011

${ }^{23}$ Tibery, C. L., and Wrench Jr. J. W., "Tables of the Goldstein Factor," David Taylor Model Basin, Rept. 1534, Applied Mathematics Laboratory, Wash. D. C., 1964.

${ }^{24}$ Foster, S. P. and Ribner, H. S., "Ideal Efficiency of Propellers Based on Theodorsen's Theory: A Review and Computer

Study, With Extended Plus Simplified Charts," University of Toronto Institute for Aerospace Studies, TN 271, Toronto, ON, Feb. 1991.

${ }^{25}$ Okulov, V. L., “On the Stability of Multiple Helical Vortices,” Journal of Fluid Mechanics, Vol. 521, 2004, pp. 319-342.

${ }^{26}$ Wald, Q. R., "The Aerodynamics of Propellers," Progress in Aerospace Sciences 42, 2006, pp. 85-128.

${ }^{27}$ Larrabee, E. E., and French, S. E., "Minimum Induced Loss Windmills and Propellers," Journal of Wind Engineering and Industrial Aerodynamics, Vol. 15, Nos. 1-3, 1983, p. 321.

${ }^{28}$ Bauer, A. B., "A New Look at Optimum Propeller Performance - Going from the Prandtl F Factor to a Vortex-Induced Downwash Analysis," AIAA-5560, 1997.

${ }^{29}$ Moschetta, J.-M. and Thipyopas C., “Optimization of a Biplane Micro Air Vehicle,” AIAA 2005-4613, June 2005 , p. 2.

${ }^{30}$ Norris, J. R., Propellers: The First and Final Explanation, Jack Norris, Northridge, 2007.

${ }^{31}$ Laitone, E. V., "Wind Tunnel Tests of Wings at Reynolds Numbers below 70 000," Experiments in Fluids, No. 23, 1997, p. 409.

${ }^{32}$ Selig, M. S., Guglielmo, J. J., Broeren, A. P., and Giguère, P., Summary of Low-Speed Airfoil Data, Vol. 1, SoarTech Publications, Virginia Beach, VA, 1995.

${ }^{33}$ Selig, M., UIUC Coordinate Database, University of Illinois at Urbana-Champaign, http://www.ae.illinois.edu/mselig/ads/coord database.html, April 2011.

${ }^{34}$ Selig, M. S., Lyon, C. A., Giguère, P., Ninham, C. N., and Guglielmo, J. J., Summary of Low-Speed Airfoil Data, Vol. 2 , SoarTech Publications, Virginia Beach, VA, 1996.

${ }^{35}$ Shyy, W. Lian, W., Tang, J., Viieru, D., and Liu, H., Aerodynamics of Low Reynolds Number Flyers, Cambridge University Press, 2008, p. 45. 
${ }^{36}$ Okamoto, M., Yasuda, K., and Azuma, A., “Aerodynamics Characteristics of the Wings and Body of a Dragonfly,” Journal of Experimental Biology, Vol. 199, 1996, pp. 281-294.

${ }^{37}$ Kunz, P. J., "Aerodynamics and Design for Ultra-Low Reynolds Number Flight," Ph.D. Dissertation, Stanford University, Stanford CA, June 2003.

${ }^{38}$ Lian, Y., and Shyy, W., "Laminar-Turbulent Transition of a Low Reynolds number Rigid or Flexible Airfoil," AIAA Journal, Vol. 45, No. 7, 2007, pp. 1501-1513.

${ }^{39}$ Uhlig, D. V., and Selig, M. S., "Post Stall Propeller Behavior at Low Reynolds Numbers,” AIAA 2008-407, Jan. 2008, p. 6.

${ }^{40}$ Hansen, K. M., and Bowman, W. J., "Aerodynamic Performance of Thin Flat Plate Airfoils with Different Maximum Camber Locations at Reynolds Numbers of 20,000, 40,000, and 60,000,” National Free Flight Symposium, No. 36, 2003, pp. 1521.

${ }^{41}$ Moore, J. P. IV, "Design of Fixed and Flapping Wing Micro Air Vehicles," $10^{\text {th }}$ International Micro Air Vehicle Competition, Provo, 2006.

${ }^{42}$ Cheng, Y.-L., "Fabrication Methods for Mesoscopic Flying Vehicle," Ph.D. Dissertation, Stanford Univ., Stanford CA, June 2001.

${ }^{43}$ Ackerman, M. and Butt, A., "Micro Air Vehicles Final Report,” Technical Report, Purdue Univ., West Lafayette, 2000, p. 16.

${ }^{44}$ Liu, Z., Moschetta, J.-M., Chapman, N., Barènes, R., and Xu, M., "Design of Test Benches for the Hovering Performance of Nano-Rotors," International Journal of Micro Air Vehicles, Vol. 2, No. 1, March 2010, pp. 17-32.

${ }^{45}$ Yeo, D., Loy, T. X., Ye, C. and Wei, A. S. Z., "Micro Propulsion Characterization System Design Project Conclusion Report," Univ. of Michigan Department of Aerospace Engineering, April 2007, p. D-1.

${ }^{46}$ Deters, R. W., and Selig, M. S., "Static Testing of Micro Propellers," AIAA 2008-6246, Aug. 2008.

${ }^{47}$ Chapman, N., Liu, Z., and Moschetta, J.-M., “U-80,” Département d'Aérodynamique, Institut Supérieur de l'Aéronautique et de l'Espace (SUPAERO), Toulouse, France, 2009 (unpublished).

${ }^{48}$ Yeo, D., Atkins, E., and Shyy, W., “Aerodynamic Sensing as Feedback for Ornithopter Flight Control,” AIAA-2011-552, Jan., 2011. 